\title{
Diagnostic Radiograph Based 3D Bone Reconstruction Framework: Application to Osteotomy Surgical Planning
}

\author{
Pavan Gamage ${ }^{1}$, Sheng Quan $\mathrm{Xie}^{1}$, Patrice Delmas ${ }^{2}$, and Wei Liang $\mathrm{Xu}^{3}$ \\ ${ }^{1}$ Department of Mechanical Engineering, The University of Auckland, New Zealand \\ ${ }^{2}$ Department of Computer Science, The University of Auckland, New Zealand \\ ${ }^{3}$ School of Engineering \& Advanced Technology, Massey University, New Zealand
}

\begin{abstract}
Pre-operative planning in orthopedic surgery is essential to identify the optimal surgical considerations for each patient-specific case. The planning for osteotomy is presently conducted through two-dimensional (2D) radiographs, where the surgeon has to mentally visualize the bone deformity. This is due to direct three-dimensional (3D) imaging modalities such as Computed Tomography (CT) still being restricted to a minority of complex orthopedic procedures. This paper presents a novel 3D bone reconstruct technique, through bi-planar 2D radiographic images. The reconstruction will be pertinent to osteotomy surgical diagnostics and planning. The framework utilizes a generic 3D model of the bone of interest to obtain the anatomical topology information. A 2D non-rigid registration is performed between the projected contours of this generic 3D model and extracted edges of the X-ray image to identify the planar customization required. Subsequently a free-form deformation based manipulation is conducted to customize the overall $3 \mathrm{D}$ bone shape.
\end{abstract}

Keywords: Osteotomy Surgical Planning, Patient-Specific Bone Customization.

\section{Introduction}

Pre-operative planning has been identified as an essential requirement for successful surgical outcomes [1]. The AO (Arbeitsgemeinschaft für Osteosynthesefragen) foundation for orthopedic research instructs on the importance of pre-operative planning through its medical training [1]. The surgeon should emerge from the planning phase with a clear idea of the patient's bone dimensions, the overall surgical approach, and if applicable, a detailed plan of the fixation. In current orthopedic cases most preoperative diagnostics and planning is conducted through 2D X-ray imaging. Twodimensional images lack significant spatial information that is present in 3D modalities. Imaging modalities such as CT have the ability to provide direct 3D volumetric images. However the use of such imaging is restricted to a minority of complex procedures due to constraints placed by cost, availability and risks posed by unwarranted detailed imaging. Thus an alternative to direct 3D imaging must be developed to augment procedures that currently rely on pure $2 \mathrm{D}$ radiographs. The work presented in this paper is motivated by this requirement, and proposes a $3 \mathrm{D}$ bone reconstruction framework from 2D radiographic images. In this paper femoral osteotomy has been 
used as the application of interest, however this work can also be applied to several other fracture reduction techniques [2].

Femoral osteotomy is a procedure used for the treatment of congenital and acquired (e.g. fracture malunion) bone deformities. Long bone deformity may consist of a combination of coronal, sagittal and oblique plane distortion. The most common of which are coronal and sagittal plane angular deformities [3]. For these cases, the planar angular and torsional misalignment of the bone axis can be identified through the anterior and lateral radiographs. However several key angles including acetabular anteversion, are not measurable through 2D imaging. Moreover, the plane on which the surgical cut has to be performed and the magnitude of the angular correction can only be accurately defined by measurements viable through $3 \mathrm{D}$ spatial visualization $[3,4]$.

The rest of the paper is structured as follows. Section two describes in detail the framework proposed by the authors and documents the current state-of-the-art research in pre-operative $3 \mathrm{D}$ anatomical reconstruction methodologies. Section three details the verifications performed on the developed algorithms and the overall results obtained.

\section{Methods}

The proposed framework to achieve 3D reconstruction is specified below. The 2D Xray images are initially processed to extract the edge points that potentially form the femur boundary. A non-rigid registration is then performed between the edges identified in the X-ray image and the projected contour points of the generic model. The identified point correspondences will next be interpolated to create a 2D planar translational field in both the anterior and lateral viewpoints. This translational field will identify the deformations required by the 3D anatomical model in the equivalent viewpoint. Finally a full 3D translational field will be created through a free-form deformation based interpolation and the 3D generic anatomical data deformed accordingly.

\subsection{Anatomical Generic Model Initialization}

The anatomical generic surface model utilized for our testing was segmented and extracted from CT scan data. Typically femur X-ray images are acquired in only the anterior and lateral viewpoints. Thus the pose (position/orientation) of the bone when imaged is standard on many femur X-ray images. The manual pose initialization will only be required if a different angle of acquisition is employed during the X-ray imaging. This initialization involves a six degree of freedom movement of the 3D model, with three translational and three rotational parameters.

Moreover, the generic model required certain automated pre-processing to identify the surface edge points that would be clearly identifiable on radiographic images. The outer contours in the anterior and lateral directions were identified through projection ray-tracing. As an example, the femoral generic model is shown in Figure 1(a) along with the outer contours. Accurate identification of as many of these edges as possible on radiographic images is vital as the customization process is driven by them, as discussed in the proceeding edge extraction section (Figure 1(b)). 


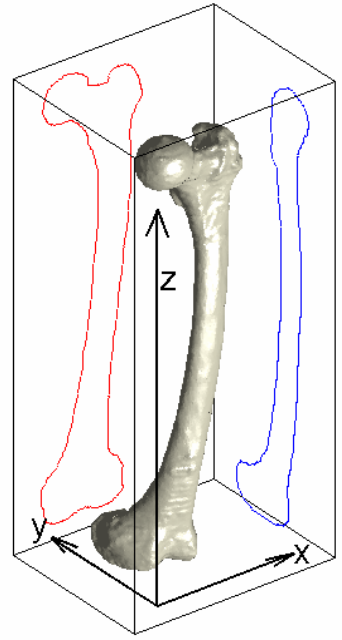

(a)
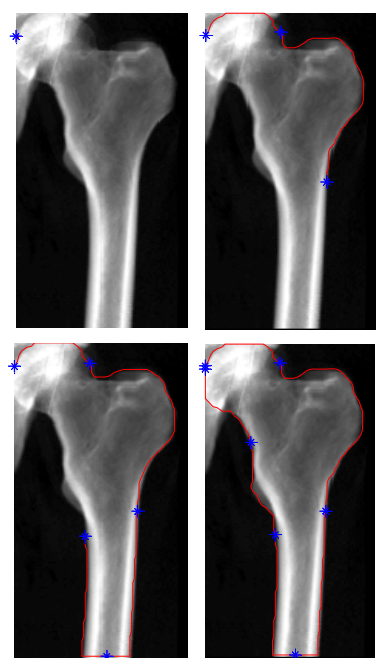

(b)

Fig. 1. Example of the clearly identifiable edges on femur radiographic images. From left to right: (a) The femoral generic model with the associated anterior and lateral projected contours; (b) Edge extraction based on an interactive LiveWire segmentation technique (detailed in section 2.2).

\subsection{Edge Extraction}

The main objective of the proposed edge extraction technique is to identify objects boundaries with sufficient continuity to be successfully employed in the proceeding shape based point correspondence estimation. Radiographic image edge extraction is hindered by poor contrast, ill defined boundaries, noise, and acquisition artifacts. Thus an interactive segmentation technique was utilized to achieve robust and accurate segmentation. The LiveWire technique proposed in this paper is based on [5] with modifications to suit grayscale X-ray image. The interactive segmentation requires the user to initially specify a seed point on the object boundary utilizing an input device (mouse). The input device must subsequently be moved to advance the cursor to a point further along the object boundary. A globally optimum path from the initial seed point to the current point is computed and displayed in real time. The optimal path is determined by assigning cost functions to boundary elements (edge strength/edge orientation), and then finding the minimum cost path. As the user moves the cursor slightly, different paths are computed and displayed. If the cursor moves close to the boundary, the LiveWire snaps to the edge. If the user is satisfied with the computed boundary segment, the cursor point can be set. This point becomes the new seed point and the recursive process continues.

The minimum cost path should correspond to an image object boundary that exhibit strong edge features. Thus, the local cost matrix is created from two edge features: gradient magnitude and gradient direction. The overall local cost matrix is computed as a weighted sum of these components. $C(m, n)$ represents the local cost of the link from pixel $m$ to a neighboring pixel $n$, where $f_{G}$ and $f_{D}$ are the gradient magnitude and gradient direction cost functions respectively and $w_{G}$ and $w_{D}$ are the corresponding scalar weights. 


$$
C(m, n)=w_{G} f_{G}+w_{D} f_{D}
$$

Gradient magnitude provides a direct correlation between edge strength and local cost. If $F$ is the grayscale $\mathrm{X}$-ray image, then the gradient magnitude $G$ is approximated with,

$$
G=\|\nabla F\|=\sqrt{\left(\frac{\partial F}{\partial x}\right)^{2}+\left(\frac{\partial F}{\partial y}\right)^{2}}
$$

The gradient is next scaled and inverted to ensure high gradients produce low costs to facilitate minimal cost path optimization.

$$
f_{G}=1-\frac{G}{\max (G)}
$$

Gradient direction adds a smoothness constraint to the boundary by associating a high cost for sharp changes in boundary direction. The gradient direction is the unit vector defined by the partials of an image $I$ in $x$ and $y\left(I_{x}\right.$ and $\left.I_{y}\right)$. The gradient direction feature cost can be defines as $f_{d}$, where $D(m)$ is the unit vector perpendicular (rotated 90 degrees clockwise) to the gradient direction at point $m$, and $n-m$ is the bidirectional link or edge vector between neighboring pixels $m$ and $n$ pointing towards $n$. Links are either horizontal, vertical, or diagonal (8-connectivity).

$$
f_{d}(m, n)=\frac{1}{\pi}[\arccos (c \cdot D(m) \cdot(n-m))+\arccos (c \cdot D(n) \cdot(n-m))]
$$

Where,

$$
\begin{gathered}
D(m)=\left(I_{y}(m),-I_{x}(m)\right), D(n)=\left(I_{y}(n),-I_{x}(n)\right) \text { and } \\
c=\operatorname{sign}(D(m) \cdot(n-m))
\end{gathered}
$$

The link direction forces high cost to an edge between two pixels that have similar gradient directions but are near perpendicular to the link between them. Thus the direction feature cost is low when the gradient directions of the two pixels are similar to each other and the link between them.

Finally, 2D graph searching is employed for an optimal minimal cost path selection. This paper utilizes the optimal graph search presented by [6]. Dijkstra's algorithm is a graph search algorithm that solves the shortest path (path with the lowest cost) problem for a graph with nonnegative edge path costs. The sequence of images in Figure 1(b) shows the interactive LiveWire segmentation technique applied to an anterior femur X-ray image.

\subsection{Shape Customization - Deformation}

A non-rigid registration between the $2 \mathrm{D}$ projective contours of the $3 \mathrm{D}$ generic model and the extracted edges of the patient-specific X-ray images is performed to customize the shape of the generic bone to that of the patient. The registration is performed through a point correspondence estimation between the two point sets. 
The similarity measure proposed in this paper to provide a measure of "correspondence" between two pairs of points or features in different images is based on shape histograms. The shape descriptor (Shape Context) describes the distribution of a series of points with respect to a given point on a shape [7]. The shape context based cost function $\left(C_{\text {shape }}\right)$ to match a point $P_{m}$ on the model contour to a point $P_{t}$ on the target contour can be expressed as (6), where $h_{m}(n)$ and $h_{t}(n)$ denote the N-bin histogram (normalized) at $P_{m}$ and $P_{t}$. Due to the chi-squared test used as the matching cost between the two shapes the $C_{\text {shape }}$ similarity measure is intrinsically bounded in $[0,1]$. The primary benefit of utilizing this measure is that it is invariant to translation, rotation, scale, and shape deformation of the two shapes under consideration.

$$
C_{\text {shape }}\left(p_{m}, p_{t}\right)=\frac{1}{2} \sum_{n=1}^{N} \frac{\left(h_{m}(n)-h_{t}(n)\right)^{2}}{h_{m}(n)+h_{t}(n)}
$$

The total cost of matching the point sets can be minimized through a Bi-partite graph searching methodology. The minimization of the total cost matrix $C_{\text {total }}$ between point sets, $P_{m}$ and $P_{t}$, is subject to the constraint that the matching is one-to-one. This square assignment problem is solved through the Hungarian algorithm with a time complexity of $O\left(\mathrm{~N}^{3}\right)$ (Figure 2(a)) [8].

The correspondences identified through the Hungarian algorithm are subsequently processed and filtered to remove any outliers (misidentified correspondences). The filtering is performed on a moving window of the identified translational values (of the correspondences) and by ensuring that they are within \pm 3 standard deviations from the local mean.

Subsequent to the planar interpolation, a 3D translational field to deform the generic model is created. The full 3D deformation is based on a cubic B-spline freeform deformation (FFD) model. FFD, introduced by [9], deforms an object by manipulating a regularly subdivided 3D parallelepiped lattice containing the object. By manipulating a mesh of control points a deformation function that specifies a new position for each point on the object is calculated. In this way the deformations of the FFD lattice are passed onto the object.

The result of FFD can be obtained from the 3D tensor product cubic B-splines, along each of the three directions, $\mathrm{U}, \mathrm{V}$ and $\mathrm{W}$, as below,

$$
T(u, v, w)=\sum_{i, j, k=0}^{3} B_{i}(u) B_{j}(v) B_{k}(w) P_{i j k}
$$

Where, $0 \leq u, v, w \leq 1$ and $B_{i}(u), B_{j}(v)$ and $B_{k}(w)$ are defined as the uniform cubic Bspline basis functions evaluated at $\mathrm{u}, \mathrm{v}$ and $\mathrm{w}$, respectively. The main objective of the FFD based deformation technique is to utilize the scattered object point translations and to configure the control grid points such that the deformed location of the selected point matches the target point location (Figure 2(b)). This is achieved in a least squares sense, based on the manipulation algorithm proposed by [10], with additions to enable multiple point movements. 


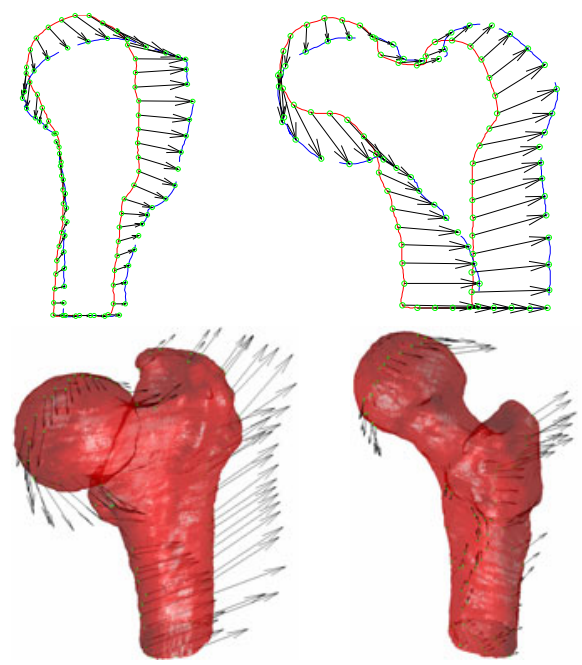

(a)
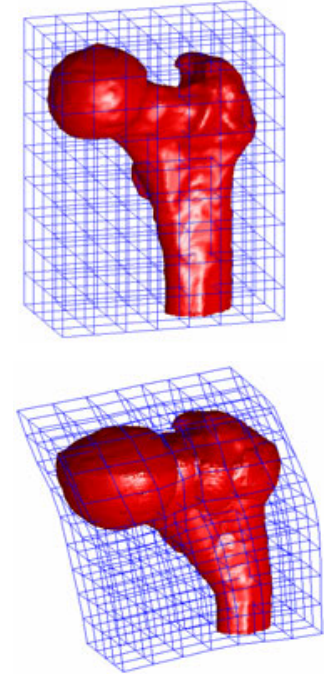

(b)

Fig. 2. Non-rigid registration performed on the edge contours and the $3 D$ translational field. From left to right: (a) Non-rigid registration on the sub-sampled edge contour data points for the anterior/lateral views (top) and the associated translational field displayed on the generic model (bottom); (b) FFD based interpolation of the transformation field (bottom) to deform the generic model (top).

\section{Results}

To validate the reconstruction framework, three cases of bone deformity were tested, femoral shaft deformity (Bowing), femoral head deformity (Shepherd's Crook), femoral condyles deformity (Erlenmeyer Flask). Two CT scans of each type of deformity and the associated X-ray images in the anterior and lateral view points were utilized in the tests. The X-ray images were used for the reconstruction and the corresponding CT scan data for accuracy assessment. Table 1 shows the quantitative results obtained from the six tests performed. Figure 3 shows several examples of the testing performed. The key accuracy measurement used during the testing is the Euclidean distance between the closest points of the reconstructed data and the ground truth CT scan data set. A discussion of the results is presented below.

Table 1. Osteotomy surgical planning 3D model reconstruction results

\begin{tabular}{cccc}
\hline $\begin{array}{c}\text { Deformity } \\
\text { Type }\end{array}$ & $\begin{array}{c}\text { Maximum Absolute } \\
\text { Error }(\mathrm{mm})\end{array}$ & $\begin{array}{c}\text { Average Absolute } \\
\text { Error }(\mathrm{mm})\end{array}$ & $\begin{array}{c}\text { Standard Devia- } \\
\text { tion }(\mathrm{mm})\end{array}$ \\
\hline Femoral Shaft & 1.31 & 0.92 & 0.093 \\
Femoral Shaft & 1.69 & 1.12 & 0.089 \\
Femoral Head & 1.86 & 0.99 & 0.093 \\
Femoral Head & 2.36 & 1.14 & 0.094 \\
Femoral Condyles & 2.05 & 1.05 & 0.089 \\
Femoral Condyles & 2.56 & 1.21 & 0.091 \\
\hline
\end{tabular}




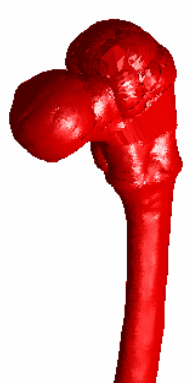

(a)

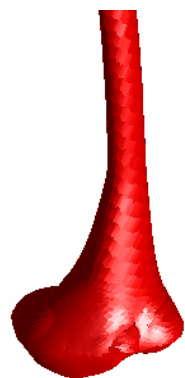

(b)

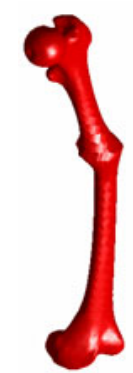

(c)

Fig. 3. Several examples of the reconstruction framework being applied. From left to right: (a) Femoral head deformity; (b) Femoral condyles deformity; (c) Femoral shaft deformity.

The results in Table 1 are within the accuracy requirements set by the authors and several other customization studies $[11,12]$. During the testing the maximum errors were clustered around the femoral condyles and head (greater and lesser trochanters). This was due to the anatomical variability in these areas.

Apart from the aforementioned areas, errors seen elsewhere are attributed to several sources.

1) The utilization of orthogonal $2 \mathrm{D}$ radiographic images for $3 \mathrm{D}$ model reconstruction is ill-posed mathematically. Making use of a-priori generic models provide crosssectional shape constraints. However there are still certain patient-specific crosssectional variations that cannot be integrated with the use of generic models. These variations cause a majority of the reconstruction errors.

2) Bone segmentation and the defining of bone contours is also a difficult task leading to reconstruction errors. Bone diameters are typically underestimated as femur $\mathrm{X}$-ray edges are blunt due to bone roundness. Femoral diameter (cortical boundary) estimation errors are typically between 0.4 to $1.0 \mathrm{~mm}$ [13]. These erroneous contours that are extracted cause inaccuracies when used for shape customization.

\section{Conclusions}

This paper introduces a bi-planar reconstruction framework which can be used for 3D osteotomy surgical planning. The authors have validated the proposed reconstruction framework, through a series of tests conducted with the aid of CT scan data. The aforementioned results exhibit the proposed method's performance in reconstruction accuracy and adaptability to several deformation types.

\section{References}

1. Schatzker, J., Tile, M.: The Rationale of Operative Fracture Care (1996)

2. Gamage, P., Xie, S.Q., Delmas, P., Xu, P.: Computer assisted 3D pre-operative planning tool for femur fracture orthopedic surgery. In: Kenneth, H.W., Michael, I.M. (eds.) SPIE, vol. 7625, p. 76253D (2010) 
3. Paley, D.: Principles of Deformity Correction. Springer, New York (2002)

4. Gunay, M.: Three-dimensional bone geometry reconstruction from X-ray images using hierarchical Free-Form Deformation and non-linear optimization. Vol. PhD dissertation. Carnegie Mellon University, Pittsburgh (2003)

5. Mortensen, E.N., Barrett, W.A.: Intelligent scissors for image composition. In: Proceedings of Computer Graphics (SIGGRAPH 1995), pp. 191-198 (1995)

6. Dijkstra, E.W.: A Note on Two Problems in Connexion with Graphs. Numerische Mathematik 1, 269-270 (1959)

7. Belongie, S., Malik, J., Puzicha, J.: Shape Matching and Object Recognition Using Shape Contexts. IEEE Transactions on Pattern Analysis and Machine Intelligence 24, 509-522 (2002)

8. Kuhn, H.W.: The Hungarian Method for the assignment problem. Naval Research Logistic Quarterly 2, 83-97 (1955)

9. Sederberg, T.W., Parry, S.R.: Free-Form Deformation of Solid Geometric Models. SIGGRAPH 20 (1986)

10. Hsu, W.H., Hughes, J.F., Kaufman, H.: Direct Manipulation of Free-Form Deformations. SIGGRAPH 26 (1992)

11. Schmutz, B., Reynolds, K.J., Slavotinek, J.P.: Customization of a generic 3D model of the distal femur using diagnostic radiographs. Journal of Medical Engineering \& Technology 32, 156-161 (2008)

12. Zheng, G., Nolte, L.P.: Surface Reconstruction of Bone from X-ray Images and Point Distribution Model Incorporating a Novel Method for 2D-3D Correspondence. In: Proceedings of the IEEE Computer Society Conference on Computer Vision and Pattern Recognition (2006)

13. Zanetti, E.M., Crupi, V., Bignardi, C., Calderale, P.M.: Radiograph-based femur morphing method. Medical and Biological Engineering and Computing 43, 181-188 (2005) 